\title{
LA MORALIDAD Y LOS VALORES DEL ALUMNADO DE "AULAS DE LA EXPERIENCIA" DE LA UNIVERSIDAD DEL PAÍS VASCO. UNA APROXIMACIÓN DESDE LA PSICOLOGÍA MORAL
}

\author{
José Domingo Gómez \\ Universidad del Pais Vasco/Euskal Herriko Unibertsitatea
}

\begin{abstract}
RESUMEN: En el presente artículo se presenta una investigación acerca del Razonamiento Moral y los Valores del alumnado de "Aulas de la Experiencia", de la Universidad del País Vasco, del campus de Álava, en Vitoria. Se han utilizado, como instrumentos de medida, tres cuestionarios: 1.- el DIT de Rest; 2.- un cuestionario elaborado específicamente de datos personales (CDP) y 3.- la Escala de Valores de Schwartz. Se han tenido en cuenta las variables sociopersonales de: género, edad, estudios realizados, posicionamiento político y creencias religiosas.

En los resultados obtenidos se observa, con significatividad estadística, que el Razonamiento Moral de este tipo de alumnado es, mayoritariamente, de rango Convencional (el $82 \%$ frente al $18 \%$ de Postconvencionalidad). Los resultados obtenidos son, en general, congruentes con las investigaciones de otros grupos de población analizada. Asimismo, aparecen también, con significatividad estadística, los Valores de: libertad, respeto, madurez, amistad, lealtad, honestidad, responsabilidad, perdón, creatividad, independencia, curiosidad, benevolencia, sentido de pertenencia.
\end{abstract}

Palabras clave: Moralidad, razonamiento moral, juicio moral, psicología moral, valores.

MORALITY AND VALUES AMONG THE STUDENTS OF THE HUMANITIES PROGRAM FOR SENIORS AT THE UNIVERSITY OF THE BASQUE COUNTRY. AN APPROXIMATION FROM MORAL PSICOLOGY

ABSTRACT: In this paper we present our research about moral reasoning and values among the students of the Humanities Program for Seniors (Aulas de la 
Experiencia) of the University of the Basque Country UPV/EHU (Alava Campus). Three questionnaires were used: 1) Rest's DIT; 2) a specific questionnaire regarding personal data (CDP); and 3) Schwartz's scale of values. The following parameters were also considered: gender, age, studies completed, political tendency and religious beliefs.

The moral reasoning of $82 \%$ of the students was conventional whereas that of the remaining $18 \%$ was postconventional. These percentages would vary slightly depending on the sociopersonal parameters considered. These results agree in general with those of other investigators. The following values were also statistically significant: freedom, respect, maturity, friendship, loyalty, honesty, responsibility, forgiving, creativity, independence, curiosity, benevolence, and sense of belonging.

Keywords: Morality, moral reasoning, moral judgement, moral psicology, values.

\section{Introducción}

\section{La Moralidad}

En los últimos años se está produciendo un aumento considerable de interés, en el campo de la psicología, por el tema de la moralidad (Fuentes, 1995). Muestra de ello son el surgimiento de centros de investigación específica de esta área, la aparición de revistas especializadas y un incremento importante de bibliografía sobre ésta temática. Existen varios enfoques en el estudio de la moralidad: el enfoque psicoanalítico, el del aprendizaje social y el cognitivo evolutivo. En esta última línea cabe destacar las investigaciones de Piaget y Kohlberg.

Kohlberg es uno de los autores más representativos del estudio de la moralidad y que tiene en la actualidad más aceptación y seguidores. A él, como al mismo Piaget, le interesaba estudiar cómo razonan las personas cuando se enfrentan a problemas o asuntos de índole moral y qué cambios se observan con la edad en el modo de concebir estos problemas.

La hipótesis central de Kohlberg es que el desarrollo moral del sujeto no se limita a la mera interiorización de las reglas sociales del ambiente, sino que el individuo mismo construye nuevas estructuras; eso sí, a partir de su interacción con el medio (Pérez Delgado y García Ros, 1991). Así, Kohlberg encontrará las características estructurales por las que atraviesa el individuo, en su razonamiento moral, a lo largo de su desarrollo evolutivo, demostrando su universalidad y proponiendo un modelo nuevo, basado en seis estadios del desarrollo de la moralidad: 1. Moralidad heterónoma; 2. Moralidad individualista; 3. Moralidad interpersonal; 4. Moralidad del sistema social; 5. Derechos humanos y moralidad del bienestar social; 6. Moralidad de principios éticos generales, prescriptivos, reversibles y universalizables. 
Asimismo Kohlberg enunciará tres niveles de la moralidad: 1. Nivel preconvencional (pre-moral); 2. Nivel convencional (se trata de un nivel de moral en conformidad a papeles convencionales); 3. Nivel postconvencional (o de principios).

TABLA 1. Niveles del desarrollo moral formulada por Kohlberg (1992).

\begin{tabular}{|c|c|}
\hline NIVELES & ESTADIOS \\
\hline \multirow{2}{*}{$\begin{array}{c}\text { Nivel I } \\
\text { (pre-convencional) }\end{array}$} & Estadio 1: moralidad heterónoma \\
\hline & Estadio 2: individualismo \\
\hline \multirow{2}{*}{$\begin{array}{c}\text { El nivel II } \\
\text { (convencional) }\end{array}$} & $\begin{array}{l}\text { Estadio 3: expectativas interpersonales mutuas } \\
\text { (relaciones y conformidad interpersonal) }\end{array}$ \\
\hline & Estadio 4: Sistema y conciencia social \\
\hline \multirow{2}{*}{$\begin{array}{c}\text { El nivel III } \\
\text { (postconvencional) }\end{array}$} & $\begin{array}{l}\text { Estadio 5: contrato social o utilidad y de derechos } \\
\text { individuales }\end{array}$ \\
\hline & Estadio 6: de principios éticos universales \\
\hline
\end{tabular}

A pesar de las críticas que este modelo ha recibido (Schwder, 1982; Simpson, 1974; Sullivan 1977; Gilligan, 1982) es el más fecundo de todos los que existen en psicología y al que no es fácil reemplazar (Pérez-Delgado 1995; Beltrán, 1992) hasta el punto de que es "la clave de bóveda en que se apoya la psicología moral actual" (Mestre y Pérez-Delgado, 1997).

Pero Kohlberg no sólo aportó una teoría acerca de la evolución y del desarrollo de la moralidad en el individuo sino que, además, ideó un instrumento de investigación a través del cual puede conocerse el proceso de razonamiento que una persona tiene para resolver dilemas morales (Hersh, Reimer y Paolitto, 1984). En 1958 elaboró la "Entrevista sobre el Juicio Moral" compuesta por tres dilemas hipotéticos (Hersh, Reimer y Paolitto, 1984). Posteriormente, Rest, basándose en la teoría de Kohlberg, creó el DIT (Defining Issues Test, 1986).

\section{Los Valores}

Asimismo, al analizar la temática de los valores, uno de los enfoques psicológicos más productivo, entendidos como criterios de comportamiento humano, es el propuesto por Rokeach (1973). La contribución de Rokeach tiene en la actualidad una continuidad en el campo de la investigación de la psicología sociocognitiva de los valores (Pérez Delgado, 1991; Molpeceres, 1994; Musitu, 1995; Palacios, 1999; Gómez, 2005).

Hay que destacar, también, el trabajo de Schwartz en la que reformuló el instrumento de medida proponiendo una nueva escala de valores, más amplia que la de Rokeach (con 56 valores), intentando así integrar la conceptualización de Rokeach con el planteamiento más culturalista, resaltando los "Dominios" de 
valores con el objetivo de desvelar la estructura que subyace en los sistemas de valores (Cuadro 1).

Cuadro 1. Estructura del sistema de valores por Schwartz y Bilsky

(Fuente: Goñi, 1996).

\begin{tabular}{|ll|}
\hline \multicolumn{1}{|c|}{ DOMINIOS } & \multicolumn{1}{c|}{ VALORES } \\
\hline $\begin{array}{l}\text { Disfrute: satisfacción de necesidades } \\
\text { físicas }\end{array}$ & $\begin{array}{l}\text { Placer, vida confortable, felicidad, ser } \\
\text { alegre }\end{array}$ \\
\hline $\begin{array}{l}\text { Logro: desarrollar habilidades en orden } \\
\text { a obtener del entorno físico y social los } \\
\text { recursos para prosperar }\end{array}$ & $\begin{array}{l}\text { Ser capaz, ser ambicioso y el } \\
\text { reconocimiento social }\end{array}$ \\
\hline $\begin{array}{l}\text { Autodirección: control efectivo de los } \\
\text { acontecimientos por encima de las } \\
\text { recompensas externas }\end{array}$ & $\begin{array}{l}\text { Ser imaginativo, ser independiente ser } \\
\text { intelectual, ser lógico }\end{array}$ \\
\hline $\begin{array}{l}\text { Conformidad: inhibición de impulsos } \\
\text { y acciones que pueden herir a otros en } \\
\text { orden a una fluida interacción social }\end{array}$ & $\begin{array}{l}\text { Ser obediente, ser educado, ser limpio, } \\
\text { ser autocontrolado }\end{array}$ \\
\hline $\begin{array}{l}\text { Prosocialidad: implicación positiva y } \\
\text { activa en favor del bienestar de los demás }\end{array}$ & Ser útil, perdonar, amar, igualdad \\
\hline $\begin{array}{l}\text { Madurez: autodesarrollo, conciencia de } \\
\text { los propios valores y redefinición de los } \\
\text { mismos para alcanzar metas }\end{array}$ & $\begin{array}{l}\text { Sabiduría, amplitud de miras, amor } \\
\text { maduro, mundo en paz, ser valiente }\end{array}$ \\
\hline $\begin{array}{l}\text { Seguridad: supervivencia física y evitar } \\
\text { amenazas a su integridad }\end{array}$ & $\begin{array}{l}\text { Armonía interior, seguridad familiar, } \\
\text { seguridad nacional, un mundo en paz }\end{array}$ \\
\hline $\begin{array}{l}\text { Poder social: búsqueda de una } \\
\text { diferenciación de status social }\end{array}$ & \begin{tabular}{l} 
(Valor ausente en el listado de Rokeach) \\
\hline
\end{tabular} \\
\hline
\end{tabular}

A pesar de todo ese esfuerzo, diversos autores concluyen que se hace necesario un nuevo modelo, para describir las diferencias culturales e ideológicas en relación a los valores (Searing, 1978; Palacios, 1999).

\section{Relación: Moralidad-Valores}

Diversos autores se han interesando en la posible relación existente entre la Moralidad y los Valores de los individuos. Se han planteado cuestiones como:

¿los diferentes estadios del razonamiento moral están asociados a los distintos grupos de valores? o, ¿tiene que ver algo el nivel del juicio o razonamiento moral con los valores que prefieren los sujetos? (Gómez, 2005)

En la actualidad se ha verificado (Feather, 1988; Orizo, 1991; Villalaín et al., 1992; Garcia Alandete y Perez Delgado, 2005; García Alandete, 2009) que la elección preferencial por ciertos valores correlaciona significativamente con los diferentes estadios del razonamiento moral. Y esto porque los di- 
ferentes tipos de valores mueven a los individuos, a través de los diferentes estadios sucesivos, hacia modos de razonamiento moral más fundamentados y universales.

En la propia teoría de Kohlberg aparecen ya distintos valores humanos básicos asociados a los 6 estadios de desarrollo de la moralidad (Kohlberg, 1984). Así:

- Los estadios 1 y 2 (nivel preconvencional) se relacionan con la evitación del castigo, la obediencia en interés propio y el beneficio personal por medio del intercambio interpersonal.

- En los estadios 3 y 4 (nivel convencional) se vive a expensas de las expectativas de los demás y se cumplen los deberes según la prescripción legal.

- Los estadios 5 y 6 (nivel postconvencional) se relacionan con el razonamiento moral fundamentado, donde personalmente se reconoce la necesidad de los derechos humanos y el respeto y dignidad de las personas.

Para Rest (1975), estos estadios se relacionan positivamente con los valores de la Escala de Valores de Rokeach (1979), relativos al pensamiento racional ("ser lógico", "la igualdad") y a la armonía personal interna ("ser equilibrado"); y, negativamente se relacionarían con los valores relativos al propio interés, la convencionalidad y la obediencia a otros en función meramente del principio de la autoridad.

El tema de las posibles interacciones entre el razonamiento moral y la preferencia por determinados valores humanos, está siendo objeto de recientes investigaciones, (Cantero y D’Ocón, 1995; Pérez Delgado y Mestre, 1993; Fuentes, 1995; Gómez, 2004).

\section{Método}

\section{Objetivos}

Los objetivos de la presente investigación son: $1^{\circ}$. Analizar el Razonamiento Moral de este tipo de alumnado relacionándolo con determinadas variables sociopersonales como género, edad, estudios realizados, clase social, posicionamiento político y creencias religiosas de los sujetos; $2^{\circ}$. Describir los Valores que poseen teniendo en cuenta las mismas variables sociopersonales y $3^{\circ}$. Indagar las posibles relaciones entre la Moralidad y los Valores de los sujetos analizados.

\section{Hipótesis}

Asimismo, nos proponemos confirmar las siguientes hipótesis: $1^{\text {a }}$. La población analizada tiene, mayoritariamente, una Moralidad de tipo convencional; $2^{\mathrm{a}}$. Se encuentran diferencias significativas, en el Razonamiento Moral, asociado con algunas variables sociopersonales como: el género, la edad, el nivel de estudios, la clase social, el posicionamiento político y las creencias religiosas de los sujetos encuestados; $3^{a}$. El aprecio relativo por determinados Dominios de valor, y sus correspondientes 
valores en la escala de Schwartz, también guarda relación estadísticamente significativa con algunas variables sociopersonales (como el género, edad, nivel de estudios, clase social, posicionamiento político y creencias religiosas). $4^{\text {a }}$. Se producen correspondencias significativas entre los índices de desarrollo del Razonamiento Moral (en el DIT de Rest) con la valoración relativa por determinados valores (de la escala de Schwartz).

\section{Participantes}

Participaron en la muestra de la investigación 171 alumnos (63 varones y 108 mujeres), de un total de 277 personas que componen el alumnado en el campus de Álava. La muestra representa el 63,89\% del total del alumnado.

\section{Instrumentos de medida y variables}

Para conseguir los objetivos propuestos se han utilizado los siguientes instrumentos de medida: $1^{\circ}$. El DIT, de Rest; $2^{\circ}$ La Escala de Valores, de Schwartz y $3^{\circ}$. El Cuestionario de Datos Personales, CDP.

Se consideraron, asimismo, las siguientes variables sociopersonales: el género, la edad, los estudios realizados, el posicionamiento político y las creencias religiosas.

\section{Procedimiento}

Los instrumentos de medida fueron cumplimentados individualmente en un tiempo aproximado de 50 minutos durante el horario de clase. A todos los sujetos se les aseguró previamente el anonimato de los cuestionarios con el fin de reducir la probabilidad del efecto de deseabilidad social. Posteriormente, y mediante el paquete estadístico SPSS 11.5, con cuya ayuda se han llevado a cabo los análisis estadísticos. Son dos los análisis a los que se han sometido los datos empíricos: la prueba chicuadrado y la prueba $\mathrm{F}$ de contraste de medias. La primera es una prueba de bondad de ajuste para probar la existencia de una diferencia significativa. En cuanto a la prueba $\mathrm{F}$ se trata de verificar si las medias son significativas.

\section{Resultados}

\section{Moralidad}

Los resultados obtenidos (ver tabla 2) indican que, mayoritariamente, la población encuestada tiene un razonamiento, estadísticamente significativo, convencional (cerca del $82 \%$ frente a un 18\% postconvencional). Estos datos obtenidos son congruentes con la hipótesis de partida (Hipótesis 1: La población analizada tiene, 
mayoritariamente, una Moralidad convencional). Asimismo, nos planteamos la hipótesis 2: Se encuentran diferencias significativas en algunas variables sociopersonales (asociadas a la moralidad), que analizamos a continuación.

Los resultados obtenidos, relacionados con el género, indican una tendencia, en el nivel postconvencional de los hombres, a ser ligeramente mayor que en las mujeres (de un 18,64\% frente al 16\%).

En la variable edad, la franja de edad 55-65 años obtiene una puntuación algo mayor, en el razonamiento postconvencional, del 20\%, frente a los de 66-80 años, que es del 15\%.

En relación con los estudios realizados, los que tienen estudios de tipo Medio obtienen unos resultados postconvencionales ligeramente mayores (el 21,05\%) que los que tiene estudios secundarios (16,13\%).

TABLA 2. Nivel de Moralidad según género, edad y estudios.

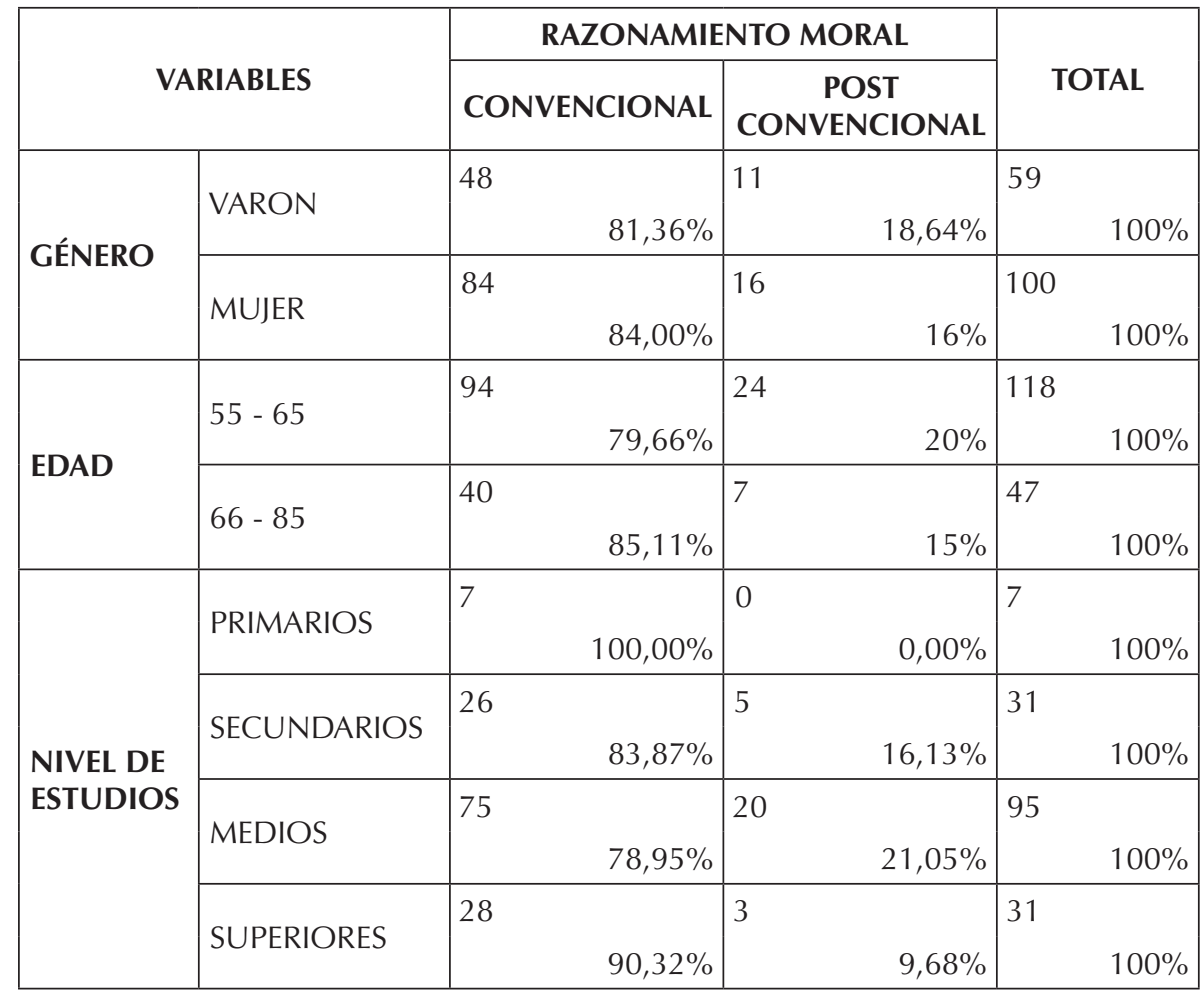

Asimismo, hemos relacionado la moralidad de los individuos con su "posicionamiento político" y sus "creencias de tipo religioso" (ver tabla 3). Los resultados han sido: que los que se manifiestan como agnósticos o indiferentes obtienen un 
razonamiento moral postconvencional mayor que los que se consideran creyentes (de un $20,83 \%$ y del $19,35 \%$ frente al 16\%). Y los que se manifiestan como "nacionalistas" o de "izquierdas" obtienen una calificación postconvencional, significativamente mayor, (de un 24,49\% y 20,83\% respectivamente) que los que se posicionan políticamente de "derechas" (el 6,90\%).

TABLA 3. Nivel de Moralidad según creencias religiosas y posicionamiento político.

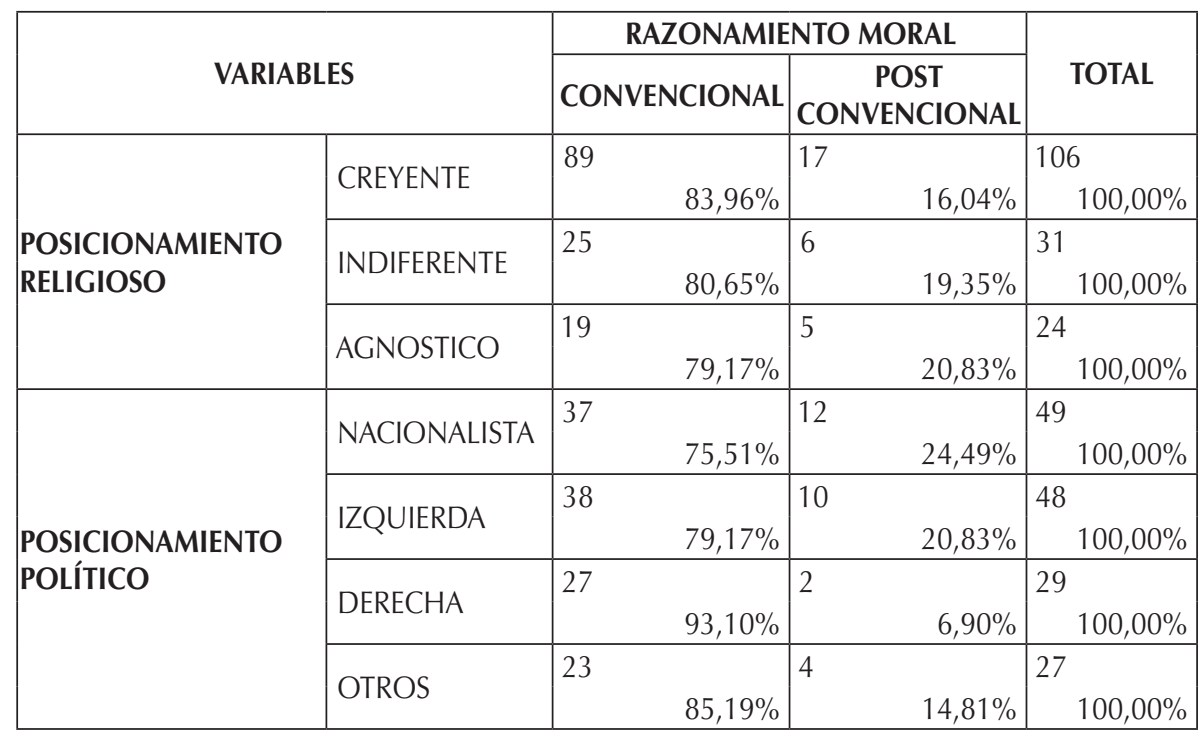

De los resultados obtenidos cabe concluir que, en general y mayoritariamente, el alumnado de Aulas de la Experiencia tiene una moralidad convencional. Que, en función de las variables tenidas en cuenta, los hombres puntúan postconvencionalmente ligeramente por encima de las mujeres. Asimismo, en los de 55-65 años, se observa una tendencia a un razonamiento postconvencional mayor. Y que los que tiene estudios de tipo medio tienden también a una moralidad postconvencional más elevada.

Y en cuanto a su posicionamiento religioso y político, los agnósticos-indiferentes obtienen puntuaciones postconvencionales mayores que los que se definen como creyentes. Asimismo, en los de izquierdas y nacionalistas se constata una puntuación postconvencional, significativamente mayor, que los que se definen como de derechas.

Todos estos resultados, en general, son congruentes con la investigación consultada previamente realizada en otros ámbitos de la población así como en otras zonas del estado español (cfr. Pérez Delgado, en la Comunidad Valenciana, 1991; Palacios, en Euskadi, 1999; y Gómez, en la UPV-EHU, 2004). 


\section{Valores}

En la temática de los valores sólo ha resultado ser, estadísticamente significativo y en relación con la variable de género, en dos Dominios concretos (ver tabla 4) preferidos por las mujeres: Autodirección (Libertad, Respeto a mi mismo, Creatividad, Independiente, Escogiendo mis metas y curioso) y Universalismo (Igualdad, Un mundo en paz, Unión con la naturaleza, Sabiduría, Un mundo de belleza, Igualdad, Abierto, Protector del medio ambiente, Triunfador con éxito). Asimismo, se detecta una tendencia, también en el grupo de las mujeres, a ser relevante el Dominio de la Benevolencia (Una vida espiritual, Sentido de pertenencia, Seguridad nacional, Amor maduro, Amistad verdadera, Leal, Honesto, Responsable, Que perdona).

La variable de la edad (tabla 5) resulta significativa en el Dominio de Autodirección (Libertad, Respeto de mi mismo, Creatividad, Independiente, Escogiendo mis metas y Curioso) pero solamente en los que tienen 55-65 años.

El Dominio de valor Universalismo (Igualdad, Un mundo en paz, Unión con la naturaleza, Sabiduría, Un mundo de belleza, Igualdad, abierto, Protector del medio ambiente, Cortesía, Triunfador con éxito); la Conformidad (Autodisciplina, Cuidadoso, Obediente, Cortesía y Fortuna) y la Seguridad (Orden social, Sentido de la vida, Reciprocidad de favores, Seguridad familiar, Saludable y Limpio) sólo ha resultado significativo en los sujetos 65-80 años.

Tabla 4. Diferencias en la preferencia por determinados dominios de valor asociados al género.

\begin{tabular}{|l|l|c|c|c|c|}
\hline \multirow{2}{*}{ DOMINIOS } & \multirow{2}{*}{ GENERO } & \multicolumn{4}{|c|}{ ESTADISTICOS } \\
\cline { 3 - 6 } & & $\mathbf{M}$ & SD & $\mathbf{F}$ & Sig. \\
\hline \multirow{2}{*}{ ESTIMULACIÓN } & Hombre & 5,39 & 1,81 & 0,51 & 0,48 \\
& Mujer & 5,61 & 1,90 & & \\
\hline \multirow{2}{*}{ AUTODIRECCIÓN } & Hombre & 7,41 & 1,22 & 3,90 & $* 0,050$ \\
& Mujer & 7,78 & 1,08 & & \\
\hline \multirow{2}{*}{ UNIVERSALISMO } & Hombre & 11,05 & 1,00 & 4,58 & $* 0,034$ \\
& Mujer & 11,36 & 0,72 & & \\
\hline \multirow{2}{*}{ TRADICIÓN } & Hombre & 6,48 & 1,35 & 1,35 & 0,25 \\
& Mujer & 6,75 & 1,38 & & \\
\hline \multirow{2}{*}{ CONFORMIDAD } & Hombre & 7,45 & 1,45 & 0,67 & 0,41 \\
& Mujer & 7,63 & 1,24 & & \\
\hline \multirow{2}{*}{ SEGURIDAD } & Hombre & 8,10 & 1,16 & 0,35 & 0,55 \\
& Mujer & 8,22 & 1,05 & & \\
\hline \multirow{2}{*}{ PODER } & Hombre & 5,20 & 1,49 & 0,17 & 0,68 \\
& Mujer & 5,31 & 1,51 & & \\
\hline
\end{tabular}




\begin{tabular}{|l|l|c|c|c|c|}
\hline \multirow{2}{*}{ DOMINIOS } & \multirow{2}{*}{ GENERO } & \multicolumn{4}{|c|}{ ESTADISTICOS } \\
\cline { 3 - 6 } & & $\mathbf{M}$ & SD & F & Sig. \\
\hline \multirow{2}{*}{ LOGRO } & Hombre & 6,92 & 1,34 & 2,48 & 0,12 \\
& Mujer & 7,26 & 1,21 & & \\
\hline \multirow{2}{*}{ HEDONISMO } & Hombre & 7,46 & 1,72 & 0,15 & 0,70 \\
& Mujer & 6,35 & 1,75 & & \\
\hline \multirow{2}{*}{ BENEVOLENCIA } & Hombre & 7,48 & 1,15 & 3,55 & 0,06 \\
& Mujer & 7,80 & 0,82 & & \\
\hline
\end{tabular}

$M=$ media $\quad S D=$ desviación típica $\quad * \mathrm{p}<, 05$

$\mathrm{F}=$ frecuencia $\mathrm{P}=$ índice Pearson

TABLA 5. Diferencias en la preferencia por determinados dominios de valor asociados a la edad.

\begin{tabular}{|c|c|c|c|c|c|}
\hline \multirow{2}{*}{ Dominios } & \multirow{2}{*}{ EDAD } & \multicolumn{4}{|c|}{ Estadísticos } \\
\hline & & $M$ & SD & $\mathbf{F}$ & Sig. \\
\hline \multirow{2}{*}{ ESTIMULACIÓN } & $55-65$ & 5,52 & 1,95 & 1,06 & 0,35 \\
\hline & $66-80$ & 5,55 & 1,63 & & \\
\hline \multirow{2}{*}{ AUTODIRECCIÓN } & $55-65$ & 7,73 & 1,08 & 4,98 & $* 0,01$ \\
\hline & $66-80$ & 7,38 & 1,34 & & \\
\hline \multirow{2}{*}{ UNIVERSALISMO } & $55-65$ & 11,20 & 0,88 & 8,41 & $* 0,00$ \\
\hline & $66-80$ & 11,25 & 0,85 & & \\
\hline \multirow{2}{*}{ TRADICIÓN } & $55-65$ & 6,67 & 1,38 & 0,82 & 0,44 \\
\hline & $66-80$ & 6,59 & 1,37 & & \\
\hline \multirow{2}{*}{ CONFORMIDAD } & $55-65$ & 7,45 & 1,37 & 2,40 & $* 0,09$ \\
\hline & $66-80$ & 7,72 & 1,26 & & \\
\hline \multirow{2}{*}{ SEGURIDAD } & $55-65$ & 8,13 & 1,15 & 4,65 & $* 0,01$ \\
\hline & $66-80$ & 8,20 & 0,97 & & \\
\hline \multirow{2}{*}{ PODER } & $55-65$ & 5,28 & 1,53 & 0,91 & 0,41 \\
\hline & $66-80$ & 5,16 & 1,34 & & \\
\hline \multirow{2}{*}{ LOGRO } & $55-65$ & 7,20 & 1,24 & 0,10 & 0,90 \\
\hline & $66-80$ & 6,98 & 1,33 & & \\
\hline \multirow{2}{*}{ HEDONISMO } & $55-65$ & 6,45 & 1,79 & 0,53 & 0,59 \\
\hline & $66-80$ & 6,18 & 1,52 & & \\
\hline \multirow{2}{*}{ BENEVOLENCIA } & $55-65$ & 7,62 & 0,97 & 2,15 & 0,12 \\
\hline & $66-80$ & 7,75 & 1,04 & & \\
\hline
\end{tabular}

$\mathrm{M}=$ media $\quad \mathrm{SD}=$ desviación típica $\quad * \mathrm{p}<, 05$

$\mathrm{F}=$ frecuencia $\mathrm{P}=$ índice Pearson 
En la variable de estudios no ha producido ninguna significatividad estadística en ninguno de los diez Dominios de valores analizados (ver tabla 6).

TABLA 6. Diferencias en la preferencia por determinados dominios de valor asociados a los estudios realizados.

\begin{tabular}{|c|c|c|c|c|c|}
\hline \multirow{2}{*}{ Dominios } & \multirow{2}{*}{ NIVEL } & \multicolumn{4}{|c|}{ Estadísticos } \\
\hline & & $M$ & SD & $\mathbf{F}$ & Sig. \\
\hline \multirow{4}{*}{ ESTIMULACIÓN } & Primarios & 5,72 & 1,77 & 0,16 & 0,92 \\
\hline & Secundarios & 5,34 & 2,00 & & \\
\hline & Medios & 5,59 & 1,76 & & \\
\hline & Superiores & 5,47 & 2,05 & & \\
\hline \multirow{4}{*}{ AUTODIRECCIÓN } & Primarios & 7,83 & 1,26 & 0,48 & 0,70 \\
\hline & Secundarios & 7,44 & 1,02 & & \\
\hline & Medios & 7,70 & 1,22 & & \\
\hline & Superiores & 7,54 & 1,04 & & \\
\hline \multirow{4}{*}{ UNIVERSALISMO } & Primarios & 11,60 & 0,75 & 0,65 & 0,59 \\
\hline & Secundarios & 11,13 & 0,77 & & \\
\hline & Medios & 11,27 & 0,90 & & \\
\hline & Superiores & 11,09 & 0,89 & & \\
\hline \multirow{4}{*}{ TRADICIÓN } & Primarios & 6,74 & 2,11 & 0,05 & 0,99 \\
\hline & Secundarios & 6,67 & 1,28 & & \\
\hline & Medios & 6,63 & 1,36 & & \\
\hline & Superiores & 6,56 & 1,43 & & \\
\hline \multirow{4}{*}{ CONFORMIDAD } & Primarios & 7,17 & 1,35 & 0,46 & 0,71 \\
\hline & Secundarios & 7,50 & 1,28 & & \\
\hline & Medios & 7,49 & 1,27 & & \\
\hline & Superiores & 7,75 & 1,59 & & \\
\hline \multirow{4}{*}{ SEGURIDAD } & Primarios & 8,77 & 0,63 & 0,59 & 0,62 \\
\hline & Secundarios & 8,07 & 1,18 & & \\
\hline & Medios & 8,14 & 1,07 & & \\
\hline & Superiores & 8,19 & 1,17 & & \\
\hline \multirow{4}{*}{ PODER } & Primarios & 5,04 & 1,46 & 0,19 & 0,90 \\
\hline & Secundarios & 5,24 & 1,49 & & \\
\hline & Medios & 5,19 & 1,49 & & \\
\hline & Superiores & 5,41 & 1,50 & & \\
\hline \multirow{4}{*}{ LOGRO } & Primarios & 6,84 & 1,37 & 0,61 & 0,61 \\
\hline & Secundarios & 7,26 & 1,24 & & \\
\hline & Medios & 7,06 & 1,35 & & \\
\hline & Superiores & 7,36 & 1,01 & & \\
\hline
\end{tabular}




\begin{tabular}{|l|l|c|c|c|c|}
\hline \multirow{2}{*}{ Dominios } & \multirow{2}{*}{ NIVEL } & \multicolumn{4}{|c|}{ Estadísticos } \\
\cline { 2 - 6 } & & $\mathbf{M}$ & SD & F & Sig. \\
\hline \multirow{4}{*}{ HEDONISMO } & Primarios & 5,92 & 1,93 & 1,53 & 0,21 \\
\cline { 2 - 6 } & Secundarios & 6,16 & 1,86 & & \\
\cline { 2 - 6 } & Medios & 6,66 & 1,63 & & \\
\cline { 2 - 6 } & Superiores & 6,02 & 1,87 & & \\
\hline \multirow{4}{*}{ BENEVOLENCIA } & Primarios & 7,56 & 1,86 & 0,21 & 0,89 \\
\cline { 2 - 6 } & Secundarios & 7,55 & 0,85 & & \\
\cline { 2 - 6 } & Medios & 7,63 & 0,97 & & \\
\cline { 2 - 6 } & Superiores & 7,75 & 1,05 & & \\
\hline
\end{tabular}

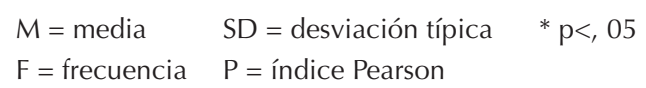

El posicionamiento político de lo sujetos analizados sí ha resultado claramente significativo (ver tabla 7) en los Dominios de valor de: Autodirección (Libertad, Respeto a mí mismo, Creatividad, Independiente, Escogiendo mis metas, curioso) y Universalismo (Igualdad, Un mundo en paz, Unión con la naturaleza, Sabiduría, Un mundo de belleza, Igualdad, Abierto, Protector del medio ambiente, Triunfador con éxito), en los sujetos que se definen "comunistas".

Al mismo tiempo, se observa una tendencia hacia la significatividad y en los Dominios de valor de Logro (Influyente, Capaz, Inteligente, Servicial) y Hedonismo (Placer, Gozar de la vida) para los que se definen igualmente "comunistas"; y en el Dominio de Tradición (Respeto por la tradición, Distanciamiento, Moderado, Devoto, Aceptar mi vida, Humilde, Respetuoso) para los que se manifiestan "nacionalistas". 
TABla 7. Diferencias en la preferencia por determinados dominios de valor asociados al posicionamiento político.

\begin{tabular}{|c|c|c|c|c|c|}
\hline \multirow{2}{*}{ Dominios } & \multirow{2}{*}{$\begin{array}{c}\text { POSICIONAMIENTO } \\
\text { POLÍTICO }\end{array}$} & \multicolumn{4}{|c|}{ Estadísticos } \\
\hline & & $M$ & SD & $\mathbf{F}$ & Sig. \\
\hline \multirow{5}{*}{ ESTIMULACIÓN } & Izq. Abertzale & 5,51 & 1,32 & 1,50 & 0,18 \\
\hline & N. Moderado & 5,77 & 1,78 & & \\
\hline & Socialista & 5,70 & 2,07 & & \\
\hline & Comunista & 8,00 & 1,00 & & \\
\hline & D. Moderada & 5,12 & 1,65 & & \\
\hline \multirow{5}{*}{ AUTODIRECCIÓN } & Izq. Abertzale & 7,49 & 0,80 & 4,71 & $* 0,000$ \\
\hline & N. Moderado & 7,64 & 1,24 & & \\
\hline & Socialista & 7,94 & 0,91 & & \\
\hline & Comunista & 9,00 & 0,58 & & \\
\hline & D. Moderada & 6,88 & 1,33 & & \\
\hline \multirow{5}{*}{ UNIVERSALISMO } & Izq. Abertzale & 11,36 & 0,69 & 3,64 & $* 0,002$ \\
\hline & N. Moderado & 11,25 & 0,67 & & \\
\hline & Socialista & 11,35 & 0,74 & & \\
\hline & Comunista & 12,23 & 0,49 & & \\
\hline & D. Moderada & 10,79 & 1,20 & & \\
\hline \multirow{5}{*}{ TRADICIÓN } & Izq. Abertzale & 6,02 & 1,01 & 2,00 & 0,07 \\
\hline & N. Moderado & 7,01 & 1,25 & & \\
\hline & Socialista & 6,62 & 1,25 & & \\
\hline & Comunista & 3,43 & & & \\
\hline & D. Moderada & 6,37 & 1,57 & & \\
\hline \multirow{5}{*}{ CONFORMIDAD } & Izq. Abertzale & 7,18 & 1,83 & 0,44 & 0,85 \\
\hline & N. Moderado & 7,60 & 1,12 & & \\
\hline & Socialista & 7,66 & 1,23 & & \\
\hline & \begin{tabular}{|l|} 
Comunista \\
\end{tabular} & 8,00 & 1,41 & & \\
\hline & D. Moderada & 7,32 & 1,78 & & \\
\hline
\end{tabular}




\begin{tabular}{|c|c|c|c|c|c|}
\hline \multirow{2}{*}{ Dominios } & \multirow{2}{*}{$\begin{array}{c}\text { POSICIONAMIENTO } \\
\text { POLÍTICO }\end{array}$} & \multicolumn{4}{|c|}{ Estadísticos } \\
\hline & & $M$ & SD & $F$ & Sig. \\
\hline \multirow{5}{*}{ SEGURIDAD } & Izq. Abertzale & 8,22 & 0,56 & 0,92 & 0,48 \\
\hline & N. Moderado & 8,12 & 0,98 & & \\
\hline & Socialista & 8,22 & 0,93 & & \\
\hline & Comunista & 8,88 & 1,44 & & \\
\hline & D. Moderada & 7,94 & 1,66 & & \\
\hline \multirow{5}{*}{ PODER } & Izq. Abertzale & 4,48 & 1,42 & 1,06 & 0,39 \\
\hline & N. Moderado & 5,54 & 1,67 & & \\
\hline & Socialista & 5,29 & 1,45 & & \\
\hline & Comunista & 5,00 & 1,24 & & \\
\hline & D. Moderada & 5,41 & 1,46 & & \\
\hline \multirow{5}{*}{ LOGRO } & Izq. Abertzale & 7,10 & 1,10 & 1,89 & 0,09 \\
\hline & N. Moderado & 7,43 & 1,44 & & \\
\hline & Socialista & 7,21 & 1,12 & & \\
\hline & Comunista & 8,40 & 1,24 & & \\
\hline & D. Moderada & 6,64 & 1,22 & & \\
\hline \multirow{5}{*}{ HEDONISMO } & Izq. Abertzale & 6,73 & 1,46 & 2,04 & 0,06 \\
\hline & N. Moderado & 6,47 & 1,55 & & \\
\hline & Socialista & 6,32 & 1,75 & & \\
\hline & Comunista & 8,88 & 0,85 & & \\
\hline & D. Moderada & 5,94 & 1,58 & & \\
\hline \multirow{5}{*}{ BENEVOLENCIA } & Izq. Abertzale & 7,54 & 0,72 & 0,35 & 0,92 \\
\hline & N. Moderado & 7,60 & 0,80 & & \\
\hline & Socialista & 7,73 & 0,90 & & \\
\hline & Comunista & 7,41 & 1,62 & & \\
\hline & D. Moderada & 7,56 & 1,33 & & \\
\hline
\end{tabular}

$M=$ media $\quad S D=$ desviación típica $\quad * p<, 05$

$\mathrm{F}=$ frecuencia $\mathrm{P}=$ índice Pearson

Finalmente, y en cuanto a las creencias religiosas que tienen los sujetos, los resultados son relevantes en cuatro Dominios de valor (tabla 8): Así, los que se definen como agnósticos o ateos priorizan el dominio Estimulación (Una vida variada, Una vida excitante, Audaz). El dominio Tradición (Respeto por la tradición), es significativa en los que se autoposicionan como creyentes y practicantes. El Dominio Logro lo priorizan los ateos-agnósticos y, finalmente, el Dominio Benevolencia es preferido por los que se definen como creyentes y practicantes. 
TABLA 8. Diferencias en la preferencia por determinados dominios de valor asociados a las creencias religiosas.

\begin{tabular}{|c|c|c|c|c|c|}
\hline \multirow{2}{*}{ Dominios } & \multirow{2}{*}{ CREENCIAS RELIGIOSAS } & \multicolumn{4}{|c|}{ Estadísticos } \\
\hline & & $M$ & SD & $F$ & Sig. \\
\hline \multirow{4}{*}{ ESTIMULACIÓN } & Creyente y Practicante & 5,51 & 1,86 & 3,39 & ${ }^{*} 0,020$ \\
\hline & Creyente no Practicante & 5,26 & 1,79 & & \\
\hline & \begin{tabular}{|l|} 
Indiferente \\
\end{tabular} & 5,29 & 2,04 & & \\
\hline & Agnóstico o Ateo & 6,61 & 1,62 & & \\
\hline \multirow{4}{*}{ AUTODIRECCIÓN } & Creyente y Practicante & 7,51 & 1,07 & 1,45 & 0,23 \\
\hline & Creyente no Practicante & 7,50 & 1,28 & & \\
\hline & Indiferente & 7.78 & 1,01 & & \\
\hline & Agnóstico o Ateo & 8,00 & 1,05 & & \\
\hline \multirow{4}{*}{ UNIVERSALISMO } & Creyente y Practicante & 11,31 & 0,78 & 0,42 & 0,74 \\
\hline & Creyente no Practicante & 11,19 & 0,99 & & \\
\hline & Indiferente & 11,10 & 0,73 & & \\
\hline & Agnóstico o Ateo & 11,32 & 0,74 & & \\
\hline \multirow{4}{*}{ TRADICIÓN } & Creyente y Practicante & 7,20 & 1,27 & 4,96 & ${ }^{*} 0,003$ \\
\hline & Creyente no Practicante & 6.65 & 1,33 & & \\
\hline & \begin{tabular}{|l|} 
Indiferente \\
\end{tabular} & 6,29 & 1,49 & & \\
\hline & Agnóstico o Ateo & 5,90 & 1,22 & & \\
\hline \multirow{4}{*}{ CONFORMIDAD } & Creyente y Practicante & 7,94 & 1,12 & 2,30 & 0,08 \\
\hline & Creyente no Practicante & 7,53 & 1,40 & & \\
\hline & Indiferente & 7,12 & 1,53 & & \\
\hline & Agnóstico o Ateo & 7,42 & 1,08 & & \\
\hline \multirow{4}{*}{ SEGURIDAD } & Creyente y Practicante & 8,06 & 1,13 & 0,39 & 0,76 \\
\hline & Creyente no Practicante & 8,13 & 1,24 & & \\
\hline & Indiferente & 8,35 & 0,90 & & \\
\hline & Agnóstico o Ateo & 8,24 & 0,84 & & \\
\hline \multirow{4}{*}{ PODER } & Creyente y Practicante & 5,18 & 1,38 & 1,29 & 0,28 \\
\hline & Creyente no Practicante & 5,52 & 1,47 & & \\
\hline & Indiferente & 5,99 & 1,44 & & \\
\hline & Agnóstico o Ateo & 4,93 & 1,73 & & \\
\hline \multirow{4}{*}{ LOGRO } & Creyente y Practicante & 7,21 & 1,26 & 2,54 & ${ }^{*} 0,058$ \\
\hline & Creyente no Practicante & 7,12 & 1,12 & & \\
\hline & Indiferente & 6,69 & 1,39 & & \\
\hline & Agnóstico o Ateo & 7,62 & 1,33 & & \\
\hline
\end{tabular}




\begin{tabular}{|l|l|c|c|c|c|}
\hline \multirow{2}{*}{ Dominios } & \multirow{3}{*}{ CREENCIAS RELIGIOSAS } & \multicolumn{4}{|c|}{ Estadísticos } \\
\cline { 3 - 6 } & & $\mathbf{M}$ & $\mathbf{S D}$ & $\mathbf{F}$ & Sig. \\
\hline \multirow{4}{*}{ HEDONISMO } & Creyente y Practicante & 6,01 & 1,62 & 2,23 & 0,09 \\
\cline { 2 - 7 } & Creyente no Practicante & 6,25 & 1,56 & & \\
\cline { 2 - 7 } & Indiferente & 6,65 & 2,12 & & \\
\cline { 2 - 7 } & Agnóstico o Ateo & 7,02 & 1,70 & & \\
\hline \multirow{4}{*}{ BENEVOLENCIA } & Creyente y Practicante & 8,09 & 0,79 & 4,42 & $* 0,005$ \\
\cline { 2 - 7 } & Creyente no Practicante & 7,61 & 1,02 & & \\
\cline { 2 - 7 } & Indiferente & 7,34 & 0,96 & & \\
\cline { 2 - 7 } & Agnóstico o Ateo & 7,35 & 0,95 & & \\
\hline
\end{tabular}

$M=$ media $\quad S D=$ desviación típica $\quad * p<, 05$

$\mathrm{F}=$ frecuencia $\mathrm{P}=$ índice Pearson

\section{Relación: Moralidad-Valores}

En los resultados obtenidos se han observado que (ver tabla 9): el nivel de razonamiento moral convencional correlaciona significativamente con los valores concretos de: Armonía interior y Una vida variada. Y el nivel postconvencional correlaciona con los valores de: Sabiduría y Ser abierto.

Asimismo, se observa una tendencia hacia la significatividad: en el nivel convencional con el valor de Reconocimiento social. La postconvencionalidad correlaciona con: Una vida espiritual, Creatividad, Un mundo de belleza y Ser audaz.

Tabla 9. Puntuaciones en los Valores y el desarrollo del Razonamiento Moral.

\begin{tabular}{|l|l|r|c|c|c|}
\hline \multicolumn{1}{|c|}{ VALORES } & NIVEL & Media & SD & F & Sig. \\
\hline \multirow{2}{*}{ Igualdad } & Conv. & 9,01 & 1,699 & 1,815 & 0,166 \\
\cline { 2 - 7 } & Post. & 9,54 & 0,744 & & \\
\hline \multirow{2}{*}{ Armonía interior } & Conv. & 9,12 & 1,498 & 3,035 & $* \mathbf{0 , 0 5 1}$ \\
\cline { 2 - 7 } & Post. & 8,36 & 2,360 & & \\
\hline \multirow{2}{*}{ Poder social } & Conv. & 3,24 & 1,717 & 0,444 & 0,642 \\
\cline { 2 - 7 } & Post. & 3,00 & 2,104 & & \\
\hline \multirow{2}{*}{ Libertad } & Conv. & 6,18 & 2,066 & 1,652 & 0,195 \\
\cline { 2 - 7 } & Post. & 6,07 & 1,811 & & \\
\hline & Conv. & 8,49 & 2,131 & 0,401 & 0,67 \\
\cline { 2 - 7 } & Post. & 8,79 & 1,292 & & \\
\hline
\end{tabular}




\begin{tabular}{|c|c|c|c|c|c|}
\hline VALORES & NIVEL & Media & SD & $\mathbf{F}$ & Sig. \\
\hline \multirow{2}{*}{ Una vida espiritual } & Conv. & 6,08 & 2,455 & 2,704 & 0,07 \\
\hline & Post. & 7,14 & 2,216 & & \\
\hline \multirow{2}{*}{ Sentido de pertenencia } & Conv. & 6,38 & 2,126 & 0,795 & 0,454 \\
\hline & Post. & 6,75 & 2,459 & & \\
\hline \multirow{2}{*}{ Orden social } & Conv. & 7,69 & 1,775 & 0,143 & 0,866 \\
\hline & Post. & 6,56 & 1,908 & & \\
\hline \multirow{2}{*}{ Una vida excitante } & Conv. & 5,22 & 2,463 & 0,182 & 0,833 \\
\hline & Post. & 5,31 & 2,480 & & \\
\hline \multirow{2}{*}{ Sentido en la vida } & Conv. & 8,12 & 1,858 & 1,984 & 0,141 \\
\hline & Post. & 8,62 & 1,678 & & \\
\hline \multirow{2}{*}{ Cortesía } & Conv. & 7,84 & 1,876 & 0,451 & 0,638 \\
\hline & Post. & 7,52 & 2,011 & & \\
\hline \multirow{2}{*}{ Fortuna } & Conv. & 5,64 & 2,091 & 1,29 & 0,278 \\
\hline & Post. & 5,14 & 2,048 & & \\
\hline \multirow{2}{*}{ Seguridad nacional } & Conv. & 6,74 & 2,728 & 0,289 & 0,749 \\
\hline & Post. & 6,39 & 2,485 & & \\
\hline \multirow{2}{*}{ Respeto a mí mismo } & Conv. & 8,41 & 1,641 & 0,147 & 0,864 \\
\hline & Post. & 8,55 & 1,270 & & \\
\hline \multirow{2}{*}{ Reciprocidad de favores } & Conv. & 7,34 & 2,182 & 0,821 & 0,442 \\
\hline & Post. & 6,79 & 2,061 & & \\
\hline \multirow{2}{*}{ Creatividad } & Conv. & 6,25 & 2,485 & 2,831 & 0,062 \\
\hline & Post. & 6,52 & 1,920 & & \\
\hline \multirow{2}{*}{ Un mundo en paz } & Conv. & 8,94 & 1,858 & 0,926 & 0,398 \\
\hline & Post. & 9,18 & 1,416 & & \\
\hline \multirow{2}{*}{ Respeto por la tradición } & Conv. & 6,36 & 2,335 & 1,605 & 0,204 \\
\hline & Post. & 5,52 & 2,668 & & \\
\hline \multirow{2}{*}{ Amor maduro } & Conv. & 7,56 & 2,083 & 1,074 & 0,344 \\
\hline & Post. & 8,18 & 1,701 & & \\
\hline \multirow{2}{*}{ Autodisciplina } & Conv. & 7,06 & 1,897 & 1,402 & 0,249 \\
\hline & Post. & 7,34 & 1,914 & & \\
\hline \multirow{2}{*}{ Distanciamiento } & Conv. & 5,89 & 2,552 & 0,082 & 0,921 \\
\hline & Post. & 6,04 & 1,644 & & \\
\hline
\end{tabular}




\begin{tabular}{|c|c|c|c|c|c|}
\hline VALORES & NIVEL & Media & SD & $\mathbf{F}$ & Sig. \\
\hline \multirow{2}{*}{ Seguridad familiar } & Conv. & 9,02 & 1,640 & 0,771 & 0,464 \\
\hline & Post. & 8,59 & 2,130 & & \\
\hline \multirow{2}{*}{ Reconocimiento social } & Conv. & 7,40 & 1,809 & 2,438 & 0,091 \\
\hline & Post. & 6,79 & 2,144 & & \\
\hline \multirow{2}{*}{ Unión con la naturaleza } & Conv. & 7,88 & 1,801 & 0,642 & 0,528 \\
\hline & Post. & 8,24 & 1,921 & & \\
\hline \multirow{2}{*}{ Una vida variada } & Conv. & 5,89 & 2,259 & 2,985 & $* 0,053$ \\
\hline & Post. & 5,55 & 2,164 & & \\
\hline \multirow{2}{*}{ Sabiduría } & Conv. & 7,78 & 1,736 & 3,081 & $* 0,049$ \\
\hline & Post. & 8,55 & 1,549 & & \\
\hline \multirow{2}{*}{ Autoridad } & Conv. & 5,01 & 2,493 & 0,862 & 0,424 \\
\hline & Post. & 4,90 & 1,934 & & \\
\hline \multirow{2}{*}{ Amistad verdadera } & Conv. & 8,57 & 1,494 & 1,068 & 0,346 \\
\hline & Post. & 9,00 & 1,069 & & \\
\hline \multirow{2}{*}{ Un mundo de belleza } & Conv. & 7,96 & 1,754 & 2,415 & 0,093 \\
\hline & Post. & 8,28 & 1,730 & & \\
\hline \multirow{2}{*}{ Justicia social } & Conv. & 9,16 & 1,240 & 1,792 & 0,17 \\
\hline & Post. & 9,55 & 1,088 & & \\
\hline \multirow{2}{*}{ Independiente } & Conv. & 7,27 & 2,010 & 0,457 & 0,634 \\
\hline & Post. & 7,72 & 1,425 & & \\
\hline \multirow{2}{*}{ Moderado } & Conv. & 7,64 & 1,815 & 0,332 & 0,718 \\
\hline & Post. & 7,48 & 2,029 & & \\
\hline \multirow{2}{*}{ Leal } & Conv. & 8,65 & 1,400 & 1,473 & 0,232 \\
\hline & Post. & 9,07 & 1,100 & & \\
\hline \multirow{2}{*}{ Ambicioso } & Conv. & 6,86 & 2,258 & 0,469 & 0,626 \\
\hline & Post. & 7,00 & 1,927 & & \\
\hline \multirow{2}{*}{ Abierto } & Conv. & 8,11 & 1,637 & 3,025 & $* 0,051$ \\
\hline & Post. & 8,93 & 1,514 & & \\
\hline \multirow{2}{*}{ Humilde } & Conv. & 6,68 & 2,096 & 0,05 & 0,952 \\
\hline & Post. & 6,61 & 2,331 & & \\
\hline \multirow{2}{*}{ Audaz } & Conv. & 5,46 & 2,282 & 2,845 & 0,061 \\
\hline & Post. & 5,93 & 2,356 & & \\
\hline
\end{tabular}




\begin{tabular}{|c|c|c|c|c|c|}
\hline VALORES & NIVEL & Media & SD & $\mathbf{F}$ & Sig. \\
\hline \multirow{2}{*}{$\begin{array}{l}\text { Protector del medio } \\
\text { ambiente }\end{array}$} & Conv. & 7,81 & 1,967 & 1,984 & 0,144 \\
\hline & Post. & 8,57 & 1,574 & & \\
\hline \multirow{2}{*}{ Influyente } & Conv. & 6,00 & 2,259 & 1,412 & 0,247 \\
\hline & Post. & 5,59 & 2,368 & & \\
\hline \multirow{2}{*}{ Respetuoso } & Conv. & 8,20 & 1,530 & 0,742 & 0,478 \\
\hline & Post. & 8,57 & 1,425 & & \\
\hline \multirow{2}{*}{ Escogiendo mis metas } & Conv. & 7,49 & 2,058 & 1,046 & 0,354 \\
\hline & Post. & 7,48 & 1,765 & & \\
\hline \multirow{2}{*}{ Saludable } & Conv. & 8,65 & 1,691 & 1,539 & 0,218 \\
\hline & Post. & 9,14 & 1,125 & & \\
\hline \multirow{2}{*}{ Capaz } & Conv. & 7,87 & 1,783 & 0,918 & 0,401 \\
\hline & Post. & 8,25 & 1,483 & & \\
\hline \multirow{2}{*}{ Aceptar mi vida } & Conv. & 7,16 & 2,404 & 0,222 & 0,801 \\
\hline & Post. & 7,10 & 2,743 & & \\
\hline \multirow{2}{*}{ Honesto } & Conv. & 8,31 & 1,625 & 0,827 & 0,439 \\
\hline & Post. & 8,50 & 1,753 & & \\
\hline \multirow{2}{*}{$\begin{array}{l}\text { Cuidadoso de mi imagen } \\
\text { pública }\end{array}$} & Conv. & 7,14 & 1,925 & 1,438 & 0,24 \\
\hline & Post. & 7,14 & 1,807 & & \\
\hline \multirow{2}{*}{ Obediente } & Conv. & 7,72 & 1,829 & 0,188 & 0,829 \\
\hline & Post. & 7,54 & 1,895 & & \\
\hline \multirow{2}{*}{ Inteligente } & Conv. & 7,47 & 1,785 & 1,161 & 0,316 \\
\hline & Post. & 8,04 & 1,710 & & \\
\hline \multirow{2}{*}{ Servicial } & Conv. & 7,39 & 1,628 & 2,851 & 0,061 \\
\hline & Post. & 8,03 & 1,783 & & \\
\hline \multirow{2}{*}{ Gozar de la vida } & Conv. & 6,44 & 2,448 & 1,995 & 0,139 \\
\hline & Post. & 6,89 & 1,771 & & \\
\hline \multirow{2}{*}{ Devoto } & Conv. & 4,91 & 3,010 & 0,265 & 0,767 \\
\hline & Post. & 5,19 & 3,397 & & \\
\hline \multirow{2}{*}{ Responsable } & Conv. & 8,35 & 1,364 & 1,587 & 0,208 \\
\hline & Post. & 8,64 & 1,129 & & \\
\hline \multirow{2}{*}{ Curioso } & Conv. & 7,52 & 1,699 & 1,063 & 0,348 \\
\hline & Post. & 7,96 & 1,753 & & \\
\hline
\end{tabular}




\begin{tabular}{|l|l|r|c|c|c|}
\hline \multicolumn{1}{|c|}{ VALORES } & NIVEL & Media & SD & F & Sig. \\
\hline \multirow{2}{*}{ Que perdona } & Conv & 7,73 & 1,757 & 0,587 & 0,557 \\
\cline { 2 - 7 } & Post. & 8,10 & 1,472 & & \\
\hline \multirow{2}{*}{ Triunfador, con éxito } & Conv & 6,09 & 2,266 & 1,564 & 0,213 \\
\cline { 2 - 7 } & Post. & 6,41 & 2,163 & & \\
\hline \multirow{2}{*}{ Limpio } & Conv & 8,08 & 2,151 & 0,719 & 0,489 \\
\cline { 2 - 7 } & Post. & 8,50 & 1,528 & & \\
\hline
\end{tabular}

$M=$ media $\quad S D=$ desviación típica $\quad * p<, 05$

$\mathrm{F}=$ frecuencia $\mathrm{P}=$ índice Pearson

\section{Conclusiones}

1. ¿Qué tipo de Moralidad tienen?

Los resultados muestran que, en general y mayoritariamente, predomina en ellos una Moralidad Convencional. Y que, en función de las distintas variables (Gráfico 1) que se han tenido en cuenta, los hombres puntúan en el nivel Postconvencional, ligeramente superior al de las mujeres. Asimismo se observa en los sujetos de 55-65 años una tendencia a tener un razonamiento Postconvencional más alto que los de 66-80. Y los que tienen realizados estudios de tipo medio tienden también a una moralidad Postconvencional ligeramente más alta.

GráfICO 1. Moralidad y Género.

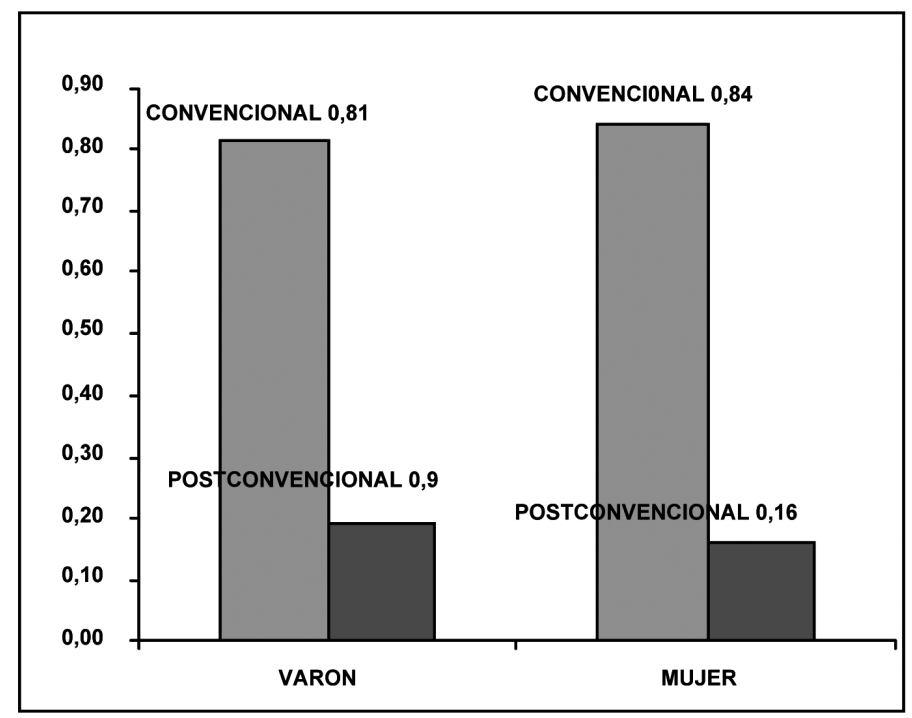


En cuanto a sus creencias religiosas y posicionamiento político: los que se declaran agnósticos-indiferentes, de izquierdas y nacionalistas, dan una puntuación postconvencional significativamente mayor que los que se definen como creyentes y de derechas (Gráficos 2 y 3 ).

GráfICO 2. Postconvencionalidad y posicionamiento político.

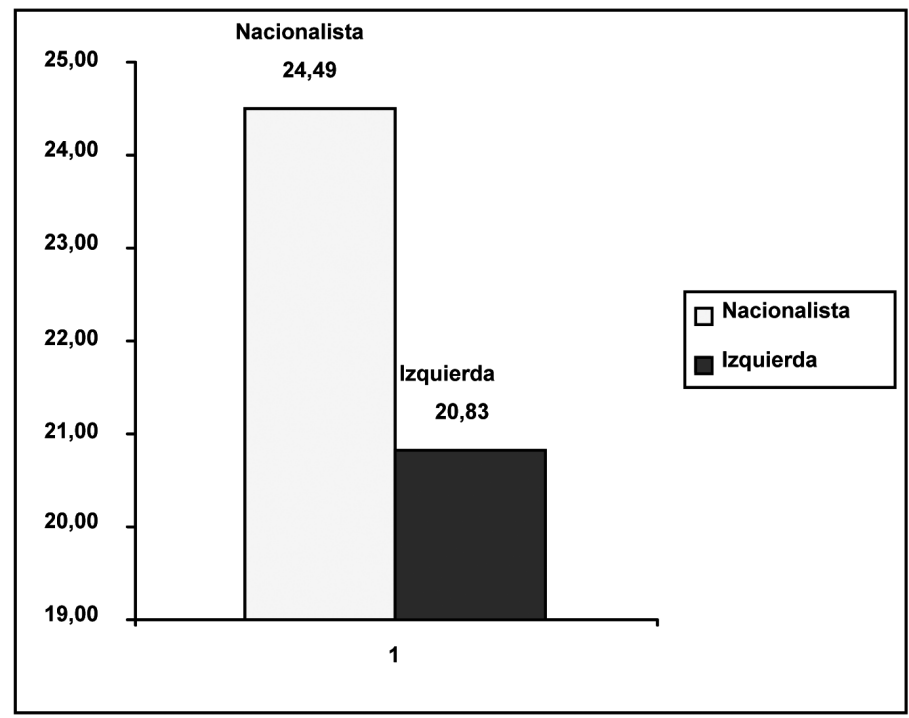

GRÁfICO 3. Postconvencionalidad y creencias religiosas.

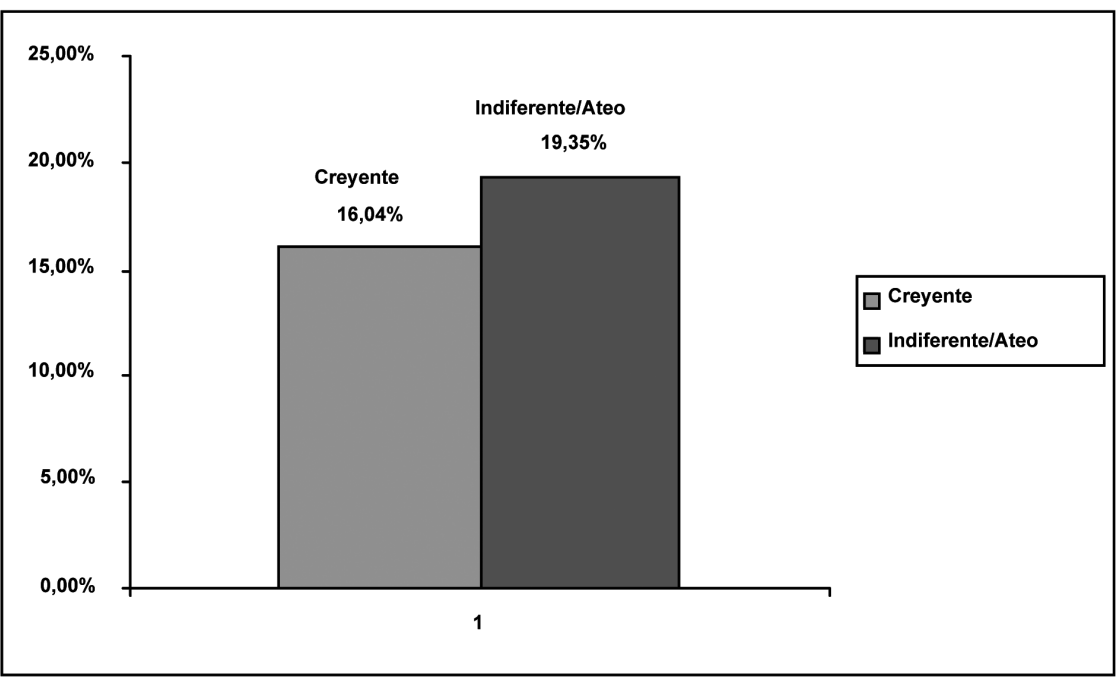




\section{2. ¿Qué Valores estiman como prioritarios?}

En relación con el género sólo ha resultado ser estadísticamente significativo, en dos Dominios concretos, preferidos por las mujeres: el dominio de Autodirección (y los valores de: Libertad, Respeto a mi mismo, Creatividad, Independiente, Escogiendo mis metas y curioso) y Universalismo (Igualdad, Un mundo en paz, Unión con la naturaleza, Sabiduría, Un mundo de belleza, Igualdad, Abierto, Protector del medio ambiente, Triunfador con éxito).

Asimismo, se observa una tendencia, también en el grupo de las mujeres, a ser relevante el Dominio de Benevolencia (con los valores de una Vida espiritual, Sentido de pertenencia, Seguridad nacional, Amor maduro, Amistad verdadera, Leal, Honesto, Responsable, Que perdona).

En cuanto a la incidencia de la edad, resulta ser estadísticamente significativa en los Dominios de Autodirección (Libertad, Respeto de mi mismo, Creatividad, Independiente, Escogiendo mis metas y Curioso) en los sujetos de 55-65 años.

En cambio en las personas de 66-80 años, son significativos los dominios de: Universalismo (Igualdad, Un mundo en paz, Unión con la naturaleza, Sabiduría, Un mundo de belleza, Igualdad, abierto, Protector del medio ambiente, Cortesía, Triunfador con éxito); la Conformidad (Autodisciplina, Cuidadoso, Obediente, Cortesía y Fortuna) y la Seguridad (Orden social, Sentido de la vida, Reciprocidad de favores, Seguridad familiar, Saludable y Limpio).

Se detecta que la variable de los estudios realizados por los sujetos, no resulta ser estadísticamente significativa en ninguno de los diez Dominios analizados.

Sin embargo, a la hora de cruzar el posicionamiento político de los individuos, sí resulta ser claramente significativo en los Dominios de valor de: Autodirección (Libertad, Respeto a mí mismo, Creatividad, Independiente, Escogiendo mis metas, curioso) y Universalismo (Igualdad, Un mundo en paz, Unión con la naturaleza, Sabiduría, Un mundo de belleza, Igualdad, Abierto, Protector del medio ambiente, Triunfador con éxito), para los sujetos que se definen políticamente como "comunistas".

Al mismo tiempo, se observa una ligera tendencia hacia la significatividad estadística, en los Dominios de valor de Logro (Influyente, Capaz, Inteligente, Servicial) y Hedonismo (Placer, Gozar de la vida) para los que igualmente se definen "comunistas". Y el Dominio de Tradición (Respeto por la tradición, Distanciamiento, Moderado, Devoto, Aceptar mi vida, Humilde, Respetuoso) resulta relevante en los que se declaran "nacionalistas".

Las creencias religiosas son relevantes en cuatro Dominios de valor. Así, los "agnósticos y ateos" valoran los dominios de Estimulación (Una vida variada, Una vida excitante, Audaz) y Logro (Influyente, Capaz, Inteligente y Servicial), frente a los "creyentes y practicantes", que valoran los dominios de Tradición (Respeto por la tradición, Distanciamiento Moderado, Devoto, Aceptar mi vida, Humilde y Respetuoso) y Benevolencia (Una vida espiritual, Sentido de perte- 
nencia, seguridad nacional, Amor maduro, Amistad verdadera, Leal, Honesto, Responsable, Que perdona).

3. ¿Se da alguna relación significativa entre Valores y Moralidad?

A este respecto, se constata en los resultados, que el nivel de razonamiento moral convencional correlaciona significativamente con los valores de: Armonía interior y Una vida variada. Y el nivel de moralidad postconvencional correlaciona con los valores de: Sabiduría y Ser abierto.

Asimismo, se observa también una tendencia hacia la significatividad. El nivel convencional correlaciona con el valor de Reconocimiento social. Y la postconvencionalidad correlaciona con Una vida espiritual, Creatividad, Un mundo de belleza y Ser audaz.

\section{Bibliografía}

BELTRÁN, J. (1992). “¿Debe defenderse a Kohlberg?". Revista Española de Pedagogía, 50, 98-112.

CANTERO, M. J. y D’OCON, A. (1995). "Experiencia profesional, desarrollo moral y valores en jóvenes trabajadores". En E. Pérez Delgado y V. Mestre, El crecimiento moral. Programas psicoeducativos y su eficacia en el aula (pp. 145-162). Valencia: Promolibro.

FEATHER, N. T. (1988). "Moral judgment and human values". British Journal of Social Psychology, 27, 239-246.

FUENTES, E. (1995). Desarrollo del juicio moral, valores y creencias en adolescentes. Tesis doctoral. Valencia: Universidad de Valencia.

GARCÍA ALANDETE, J. (2009). Actitudes religiosas. Valores y Razonamiento moral. Universidad de Valencia.

GARCÍA ALANDETE, J. y PÉREZ DELGADO, E. (2005). "Razonamiento Moral y Valores. Estudio de sus relaciones en un grupo de universitarios españoles". Revista Latinoamericana de Psicología, 37/1, 131-148.

GILLIGAN, C. (1982). "In a different voice, women's conceptions of the self and morality". Harvard Educational Review, 47, 481-517.

GÓMEZ ROZAS, J. D. (2004). Religiosidad, Moralidad y Valores de los jóvenes universitarios del campus de Álava. Tesis doctoral. Bilbao.

HERSH, R., REIMER, J. y PAOLITTO, D. (1984). El crecimiento moral de Piaget a Kohlberg. Madrid: Narcea.

KOHLBERG, L. (1992) 1984. Psicología del desarrollo moral. Bilbao: Desclée de Brouwer. 
MESTRE, V. y PÉREZ DELGADO, E. (1997). Cognición y afecto en el desarrollo moral. Evaluación y programas de intervención. Valencia: Promolibro.

MOLPECERES, M. A. (1994). El sistema de valores. Su configuración y su socialización familiar en la adolescencia. Tesis doctoral. Valencia: Universidad de Valencia.

MUSITU, G. (1995). "Familia, identidad y valores". Infancia y Sociedad, 30, 7598.

ORIZO, F. A. (1991). Los nuevos valores de los españoles. Madrid: S.M.

PALACIOS, S. (1999). La desobediencia civil y la objeción de conciencia. Comprensión sociomoral de sucesos complejos durante la adolescencia y la juventud. Tesis doctoral, Universidad País Vasco.

PÉREZ DELGADO, E. (1995). Psicología, ética, religión. Madrid: Siglo XXI.

PÉREZ DELGADO, E. y GARCÍA ROS, R. (1991). La psicología del desarrollo moral. Historia, teoría e investigación actual. Madrid: Siglo XXI.

PÉREZ-DELGADO, E. y MESTRE ESCRIBA, V. (1993). "Desarrollo del juicio moral y valores humanos". Cuadernos de Trabajo Social, 6, 61-87.

REST, J. (1979a). Development in judging moral issues. Minneapolis: University of Minnesota Press.

REST, J. (1986). DIT Manual. Manual for the Defining Issues Test. Minneapolis: University of Minnesota Press.

ROKEACH, M. (1973). The nature of human values. New York: Free Press.

ROKEACH, M. (1979). Understanding human values. Individual and societal. New York: Free Press.

SHWEDER, R. (1982). "Beyond self-constructed Knowledge: The study of culture and morality". Merrill-Palmer Quarterly, 28, 41-69.

SEARING, D. (1978). "Measuring politicians' values: Administration and assesment of a ranking technique en the British House of Commons". American Political Science Review, 72, 65-79.

SIMPSON, E. I. (1974). "Moral development research: A case study of scientific cultural bias". Human Development, 17, 81-106.

SULLIVAN, E. U. (1977). "A study of Kohberg's structural theory of moral development. A critique of liberal Social Sciencie Ideology". Human Development, 20, 352-376.

VILLALAIN, J. L., BASTERRA, L. y DEL VALLE, J. M. (1992). La sociedad española de los 90 y sus nuevos valores. Madrid: S.M. 